\title{
The ATLAS SemiConductor Tracker
} Operation and Performance

\author{
Joleen Pater \\ The University of Manchester \\ On behalf of the ATLAS Collaboration \\ PSD9 - Aberystwyth - 13 September 2011
}




\section{The ATLAS SemiConductor Tracker}
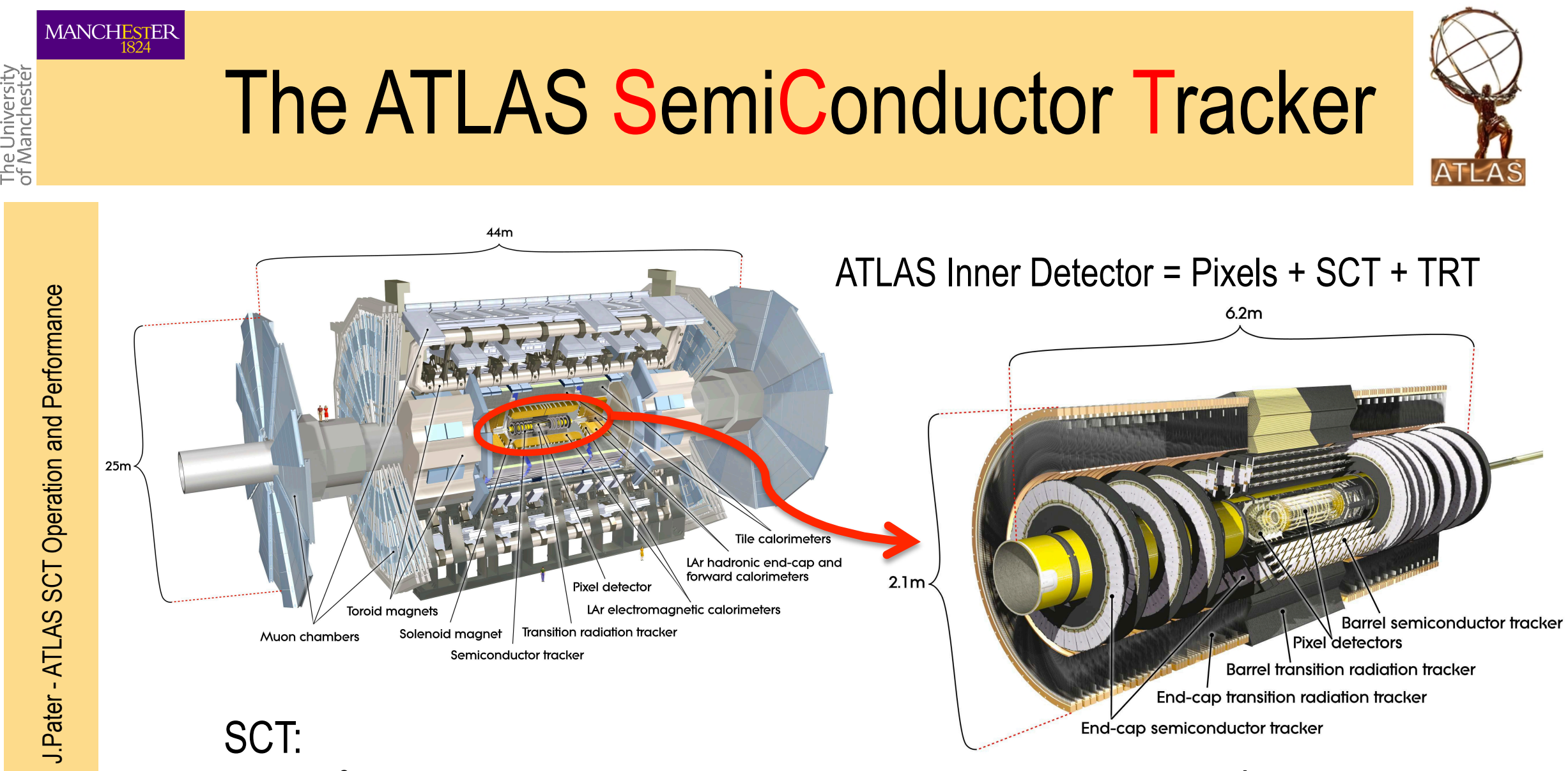

- $61 \mathrm{~m}^{2}$ of silicon strips: $30 \mathrm{~cm}<\mathrm{R}<52 \mathrm{~cm}$, hermetic coverage for $|\eta|<2.5$

- Immersed in 2-T solenoidal B field

- Barrel: 2112 rectangular modules on 4 layers

- Endcaps: 1976 wedge-shaped modules on 18 disks (9 each end)

$\rightarrow$ Tracks with $p_{T}>1 \mathrm{GeV} / c$ pass through at least 4 layers

- Total of 6.3 million readout channels

- Cooled to $-7^{\circ} \mathrm{C}$ with $\mathrm{C}_{3} \mathrm{~F}_{8}$ 


\section{SCT Modules}

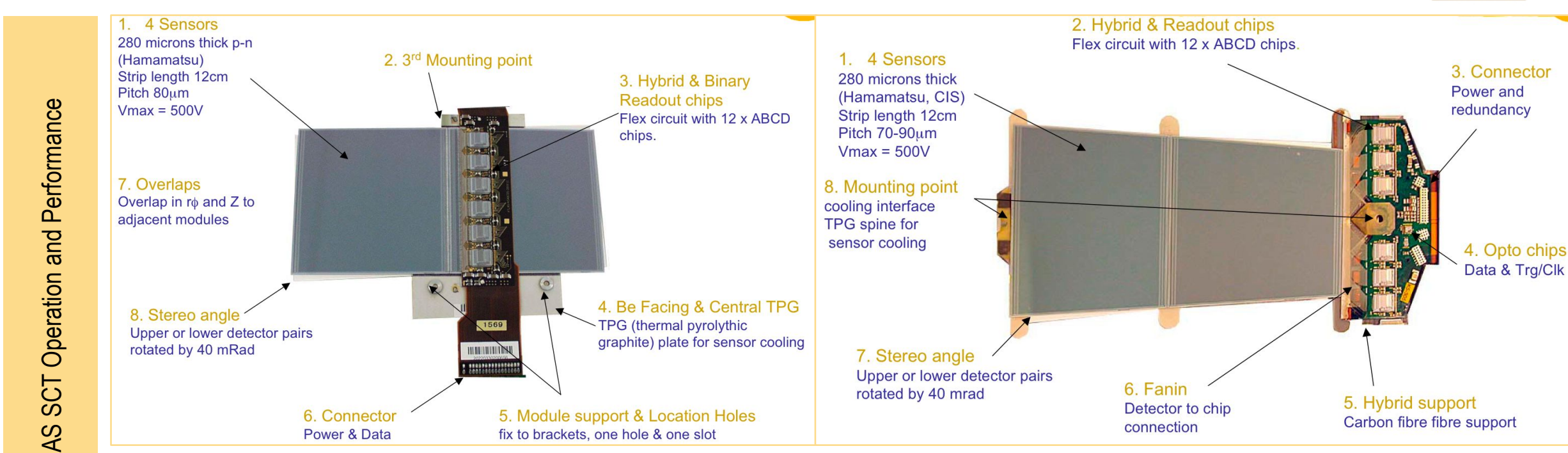

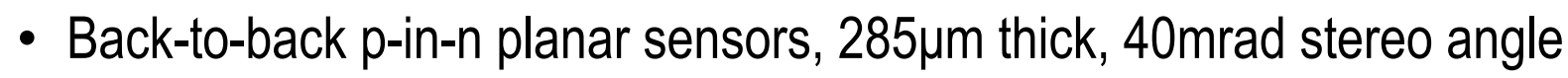

- Glued to thermally-conductive baseboard

- Bias voltage 150-500V ( $65 \mathrm{~V} / \sim 85 \mathrm{~V}$ depletion Hamamatsu/CiS)

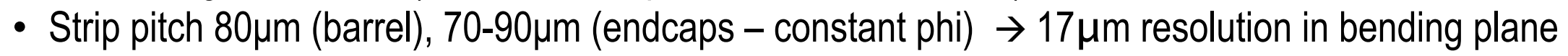

- Binary readout via ABCD3TA

- Rad-hard DMILL technology

- 12 ASICs per module, mounted on Cu-kapton flex hybrid

- Optical communication with off-detector readout electronics:

- 1 TX (clock/command) fibre, 2 RX (data) fibres per module

- Redundancy between neighbor modules.

- 4 types of endcap modules for hermetic coverage of disks 


\section{Operational History}

- Commissioned from 2007 with cosmic rays

- First LHC collisions at $\sqrt{ } \mathrm{s}=900 \mathrm{GeV}$ December 2009

- $\sqrt{ } \mathrm{s}=7 \mathrm{TeV}$ collisions in March 2010

- Increased LHC luminosity in 2011
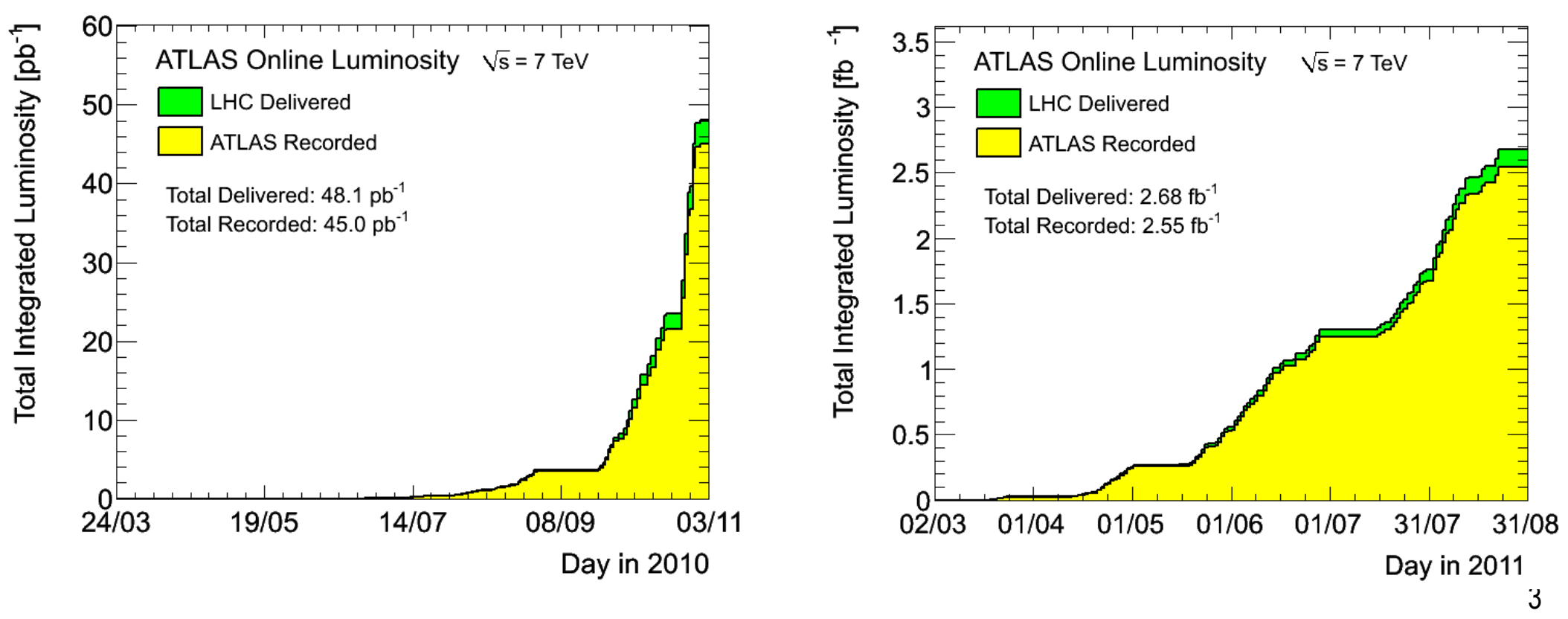


\section{SCT Status}

\section{- $>99 \%$ operational channels}

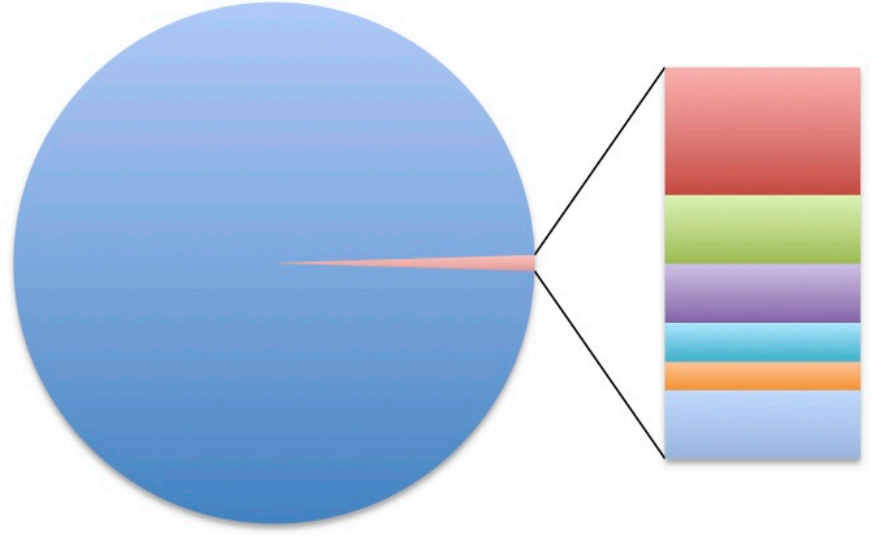

- Operational Channels

- 13 modules on a faulty cooling loop

7 modules with LV problems

-6 modules with HV problems

4 modules with readout problems

Disabled Chips

- Masked Strips

- Stable since 2009

- Operational channels are $99.9 \%$ efficient:

\begin{tabular}{|c|c|c|c|c|c|c|c|c|c|c|c|c|}
\hline \multicolumn{3}{|c|}{$\begin{array}{l}\text { Inner Tracking } \\
\text { Detectors }\end{array}$} & \multicolumn{4}{|c|}{ Calorimeters } & \multicolumn{4}{|c|}{ Muon Detectors } & \multicolumn{2}{|c|}{ Magnets } \\
\hline Pixel & SCT & TRT & $\begin{array}{l}\text { LAr } \\
\text { EM }\end{array}$ & $\begin{array}{l}\text { LAr } \\
\text { HAD }\end{array}$ & $\begin{array}{l}\text { LAr } \\
\text { FWD }\end{array}$ & Tile & MDT & $\mathrm{RPC}$ & $\mathrm{CSC}$ & TGC & Solenoid & Toroid \\
\hline 99.9 & 99.9 & 100 & 90.0 & 91.3 & 94.8 & 98.2 & 99.5 & 99.7 & 99.9 & 99.6 & 99.6 & 99.4 \\
\hline
\end{tabular}




\section{SCT DAQ stability}

- 'Warm start' procedure:

- Bias at 50V standby; near depletion so fully efficient

- To nominal $(150 \mathrm{~V})$ when beams are stable

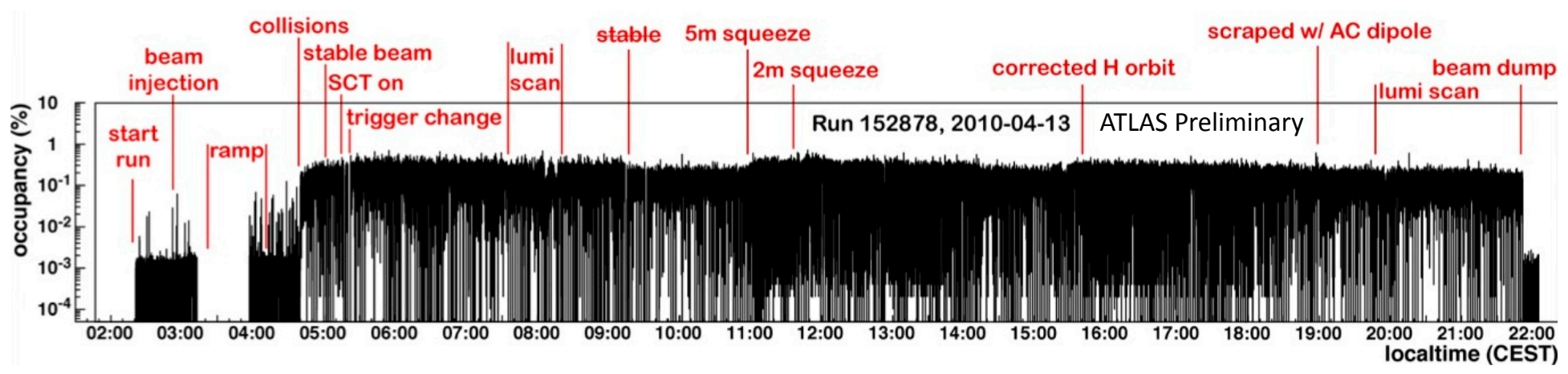

- Consistently low error rates

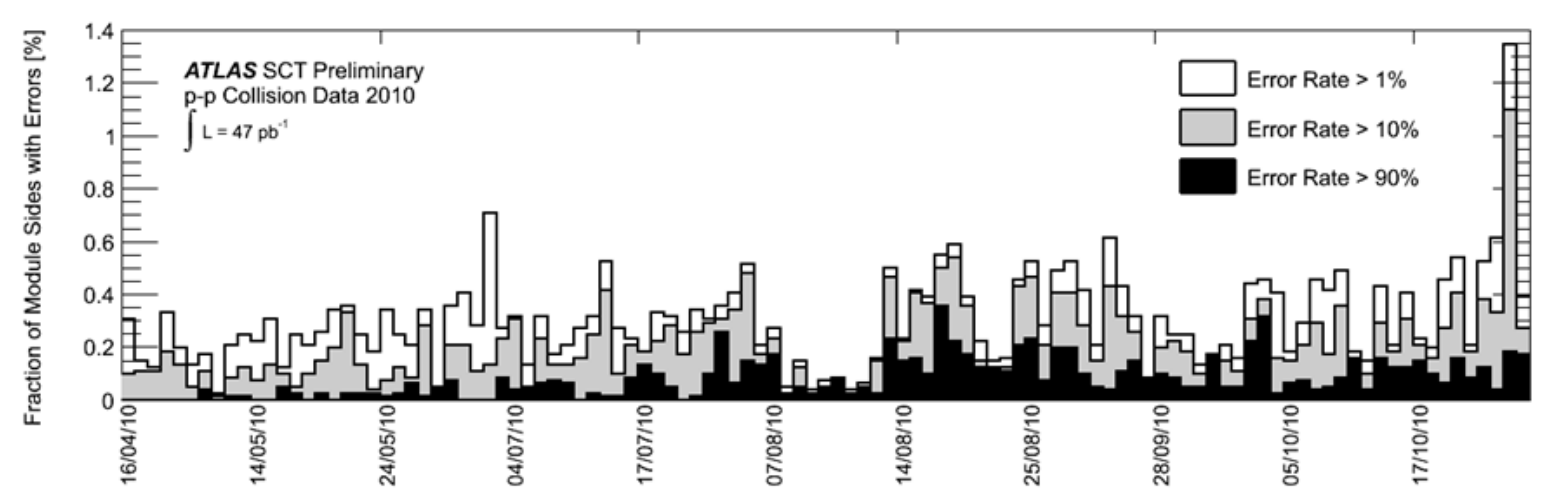




\section{Optical Transmitter Problems}

- 'TX plugin' = optical transmitter unit for RODs (off-detector)

- Holds 12 VCSELS

- In 2008 these VCSELs begin dying

- ESD damage during manufacture

- sensitivity to humidity

- Peak failure rate was $\sim 3 /$ day

- Temporary fixes:

- Utilise module redundancy

- Replace plugins with spares

- Flush crates with dryer air

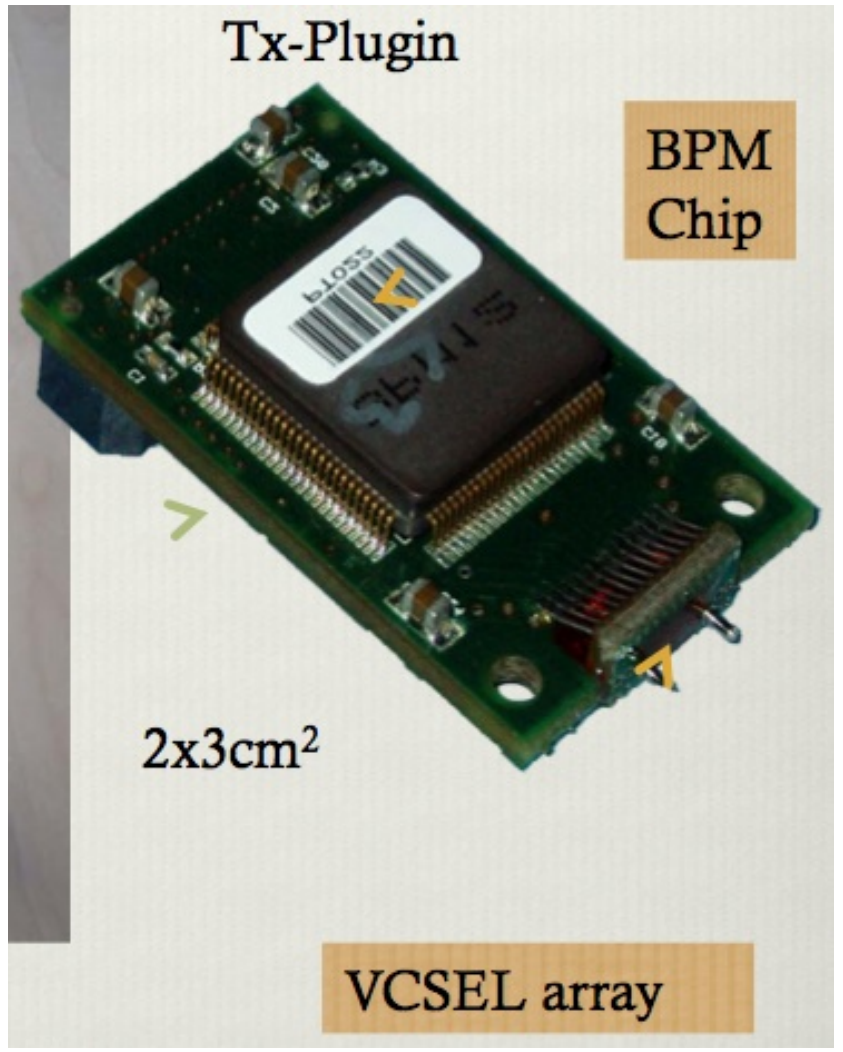

- Long term fix: replacing plugins with new units with better humidity resistance:

- 3/8 crates have new units installed

- Rest will be replaced by end of 2011 


\section{Timing}

- Three $25 n$ s time bins are read out around the trigger time

- We want the hit to be in the middle bin

- Early running: "XXX" = accept a hit in any of the 3 bins

- Now: "X1X" = require a hit in the correct bin

- Future: "01X"

- Timing scans are done for each module to ensure this

- Adjustments made for fibre lengths, time-offlight from IP
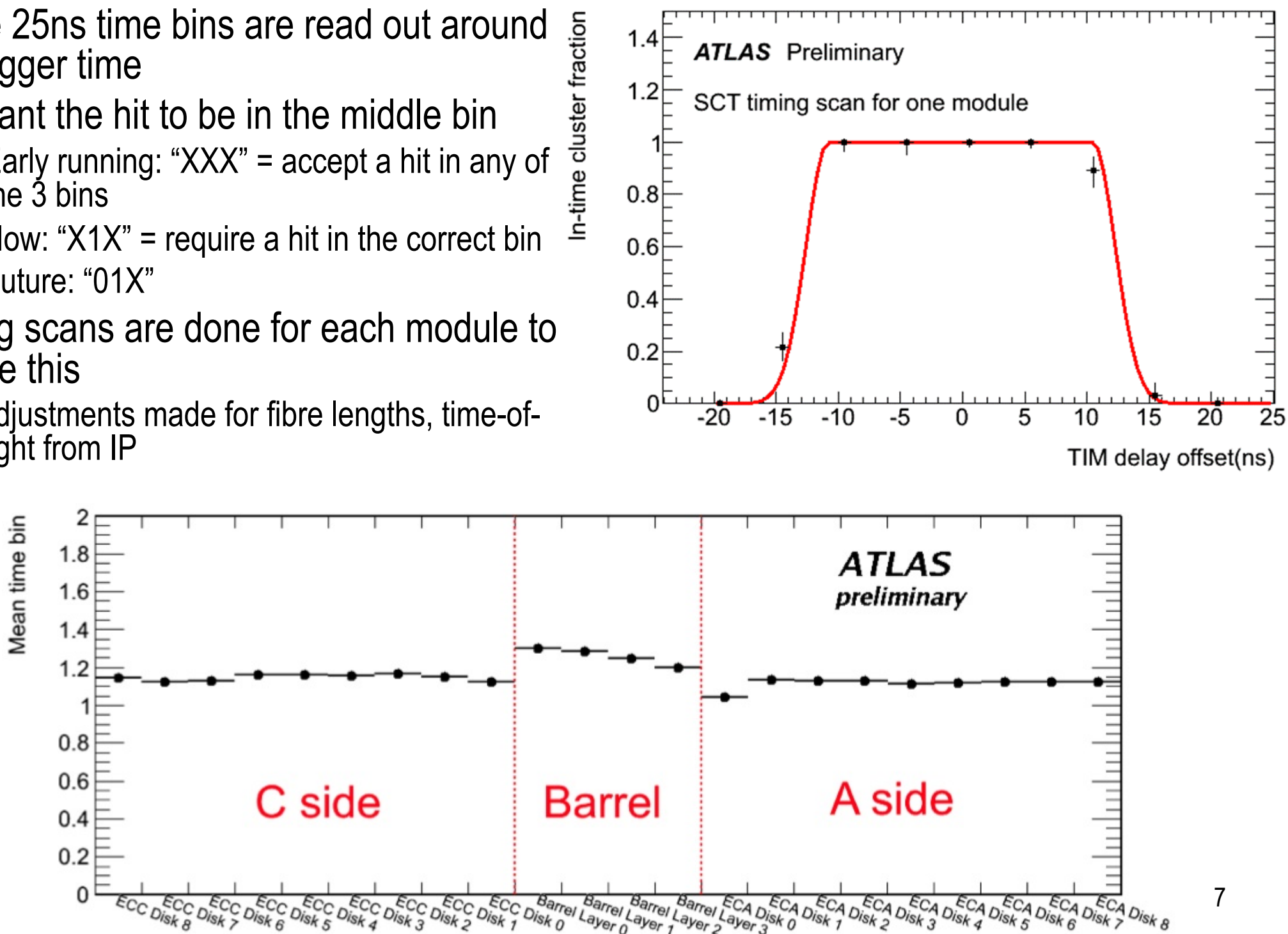


\section{Noise Occupancy}

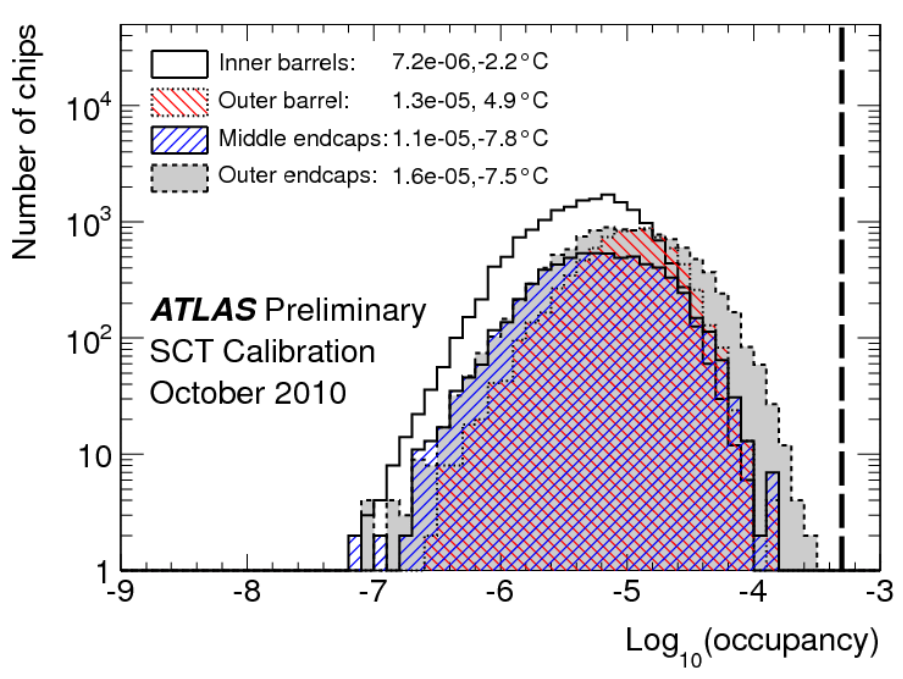

- Noise measured two ways:

- Calibration pulses injected by the ASIC $\rightarrow$ s-curve fits give measure of noise

- Measure noise occupancy as a function of threshold

$\rightarrow$ Extract input noise

- Results are consistent and below specification:

Occupancy $<5 \times 10^{-4}$, noise $<1500$ e-
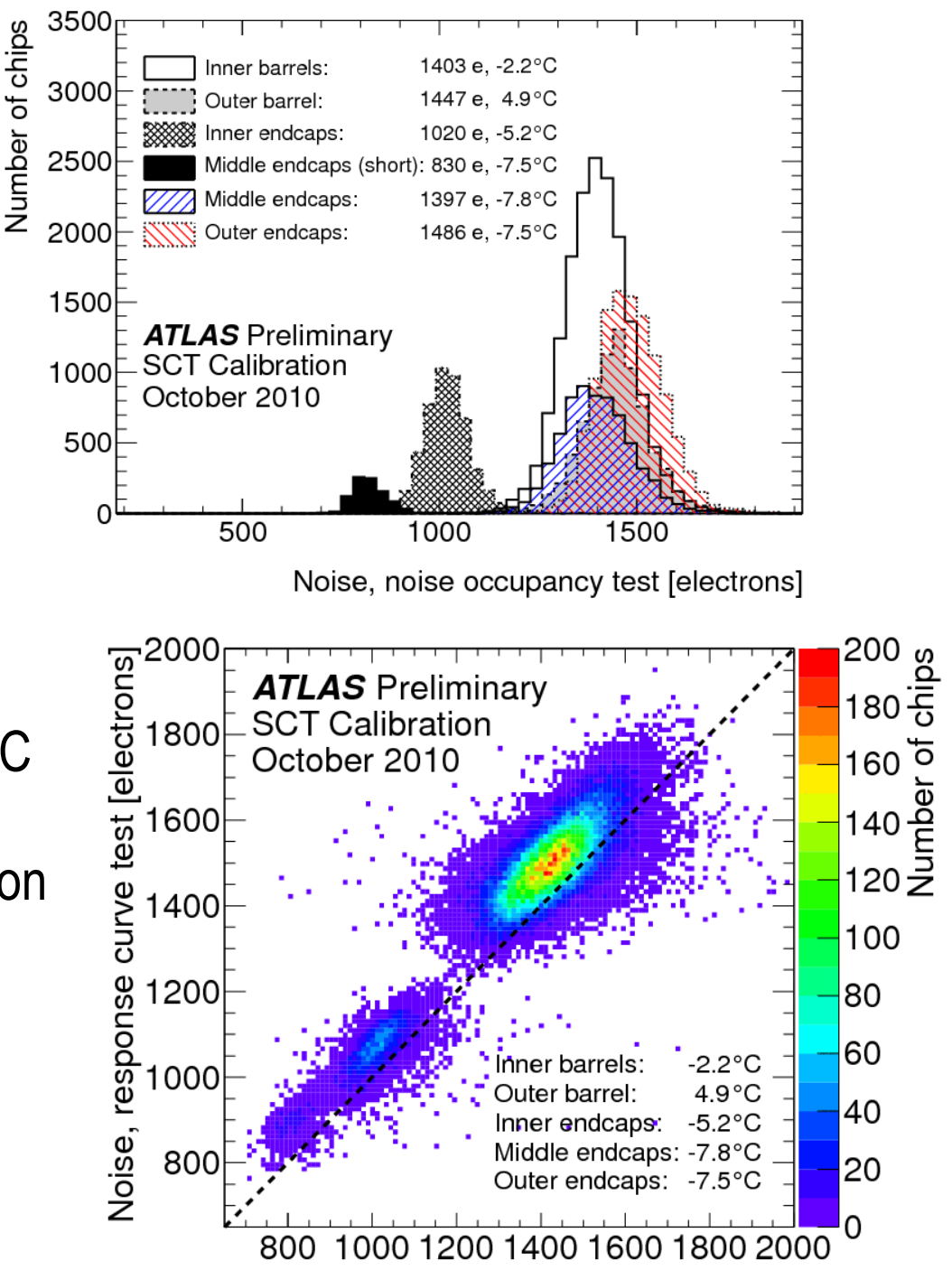

Noise, noise occupancy test [electrons] 


\section{Strip Occupancy}

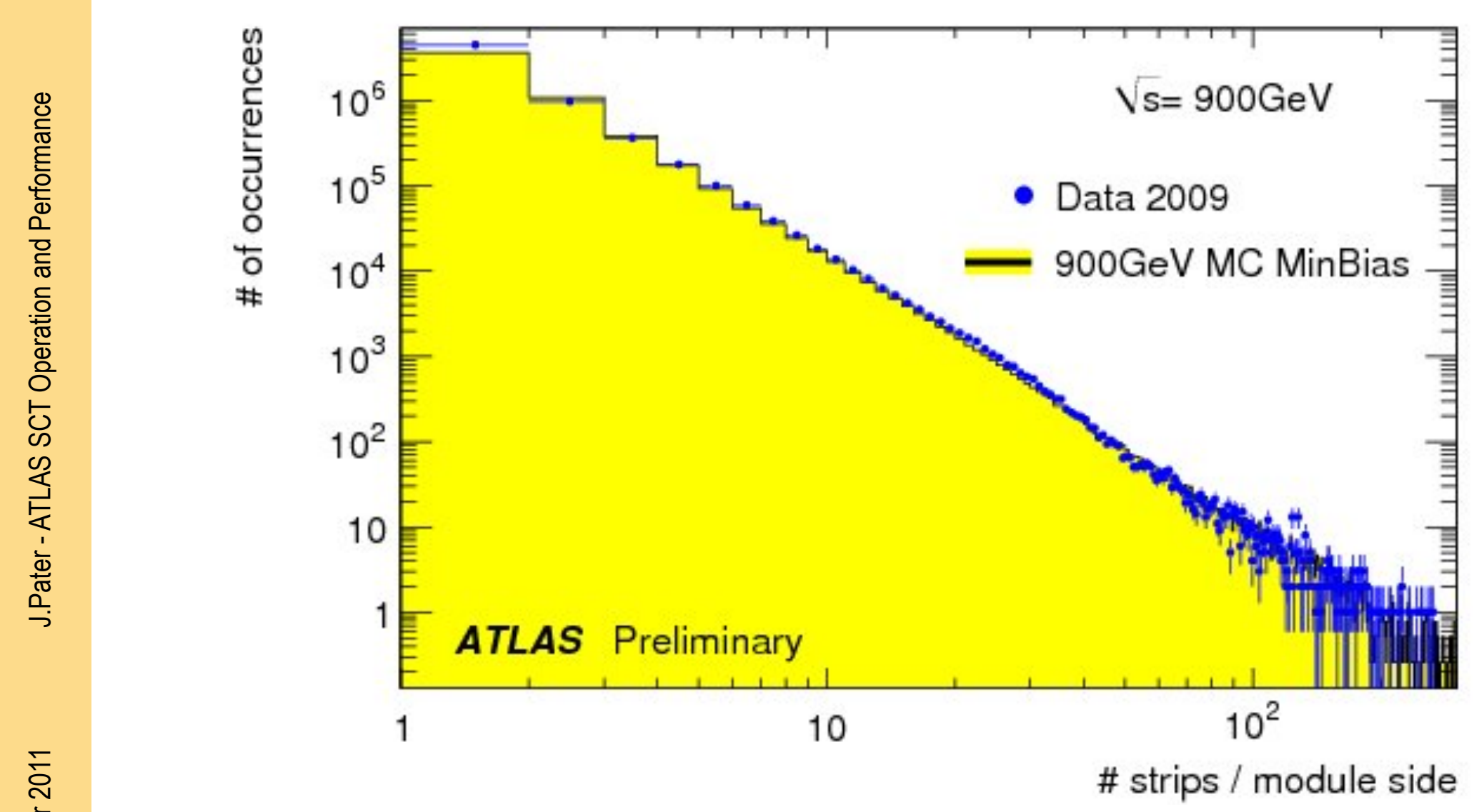

- Plot shows number of strips per module side in a $900-\mathrm{GeV}$ run compared to simulation

- Good agreement MC-data 


\section{Hit Efficiency from Tracks}

Plots show number of hits / number of possible hits

(dead modules/chips removed) Track requirements:

- $\mathrm{pT}>1 \mathrm{GeV} / \mathrm{c}$

- $>7$ SCT hits for standalone tracks

- >6 SCT hits for combined tracks
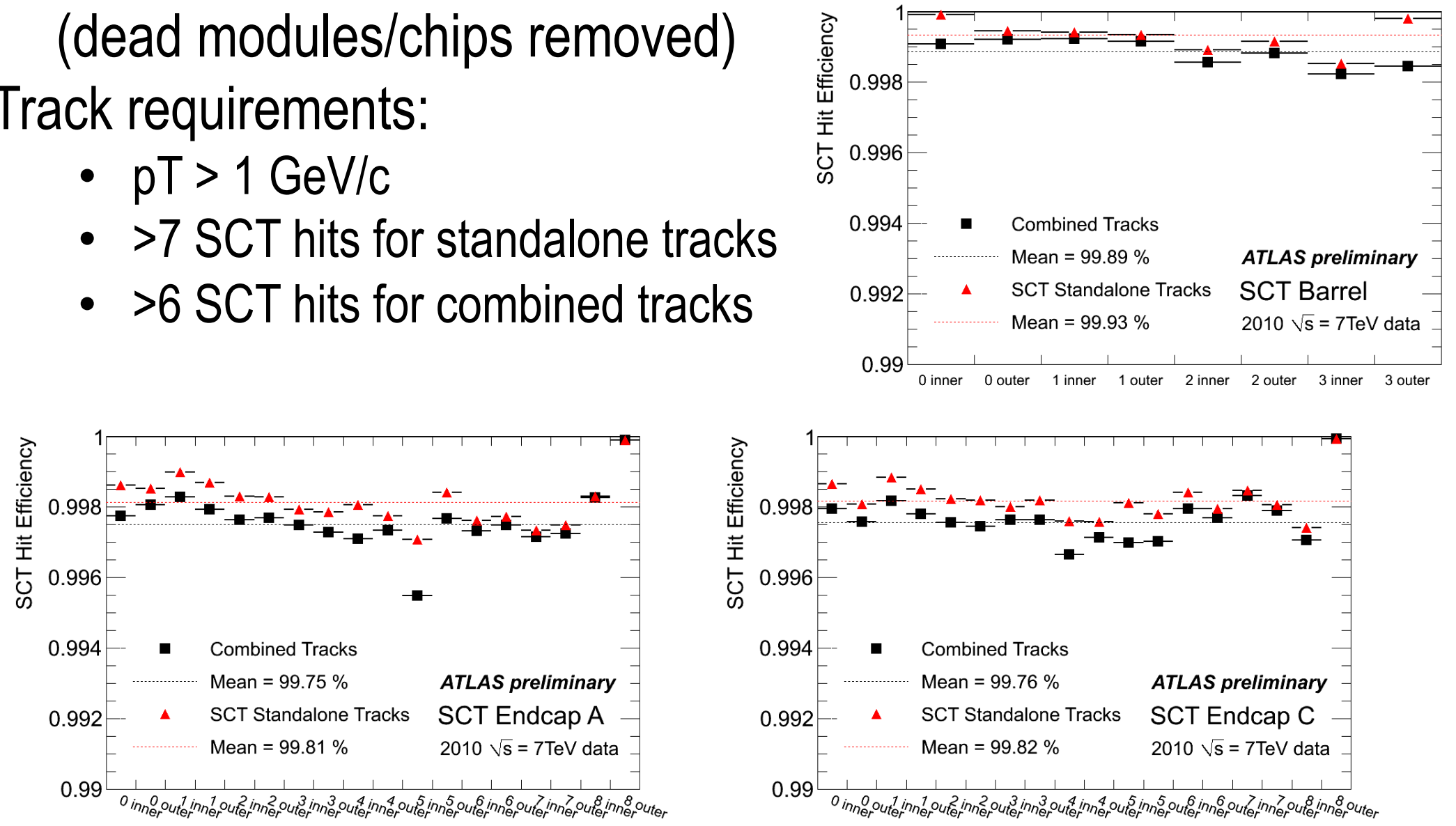

Much better than specification of $>99 \%$ 


\section{Mechanical Stability and Alignment}

Frequency Scanning Interferometry shows:

- Stability to $\ll 1 \mu$ m during runs

- Shifts of 3-5 $\mu \mathrm{m}$ during e.g. temperature, B-field changes

$X^{2}$ minimisation of track-to-hit residuals:
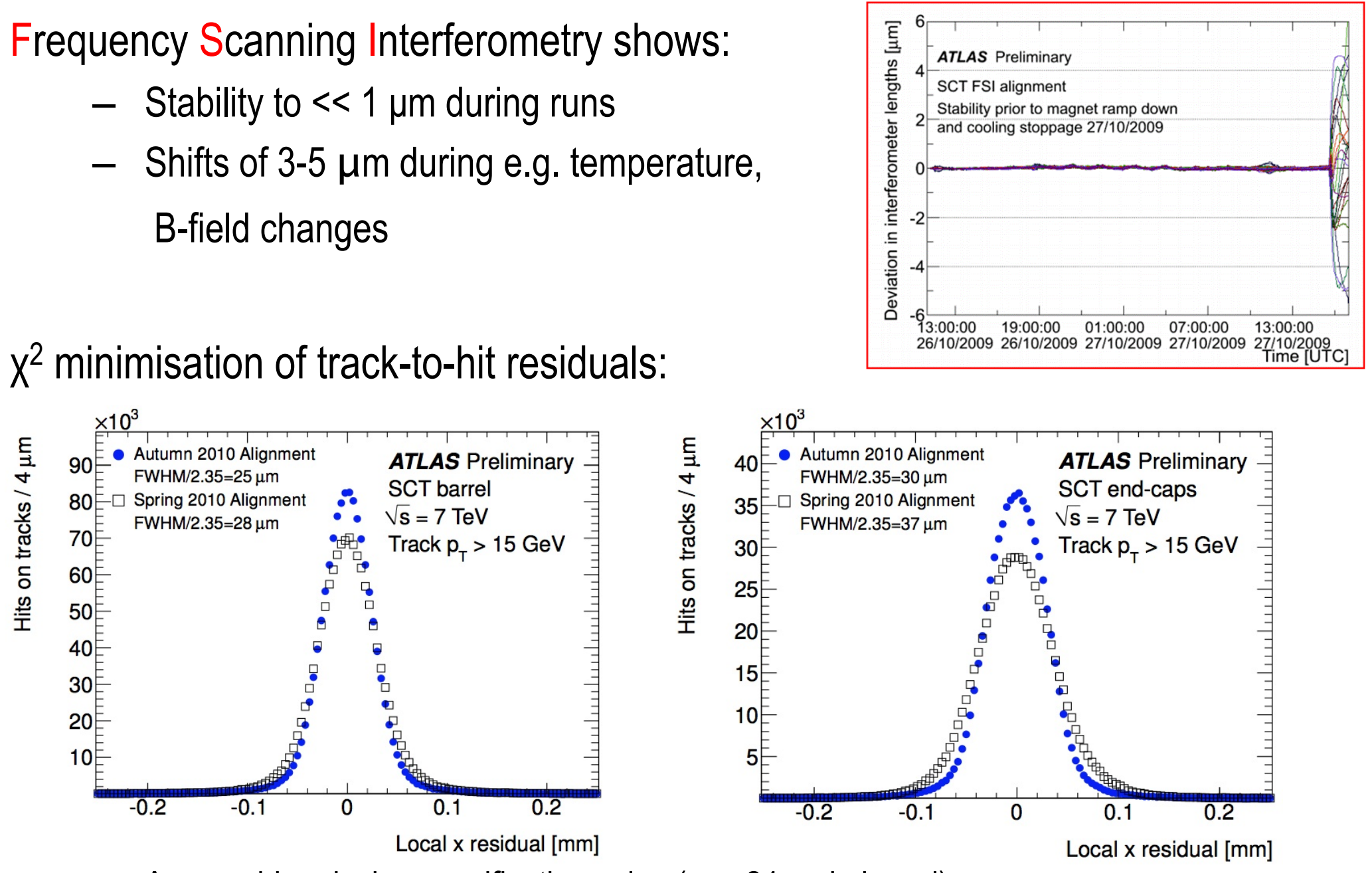

- Approaching design specification value (e.g. $24 \mu \mathrm{m}$ in barrel)

- Time-dependent studies will become possible with more statistics 


\section{Lorentz Angle}

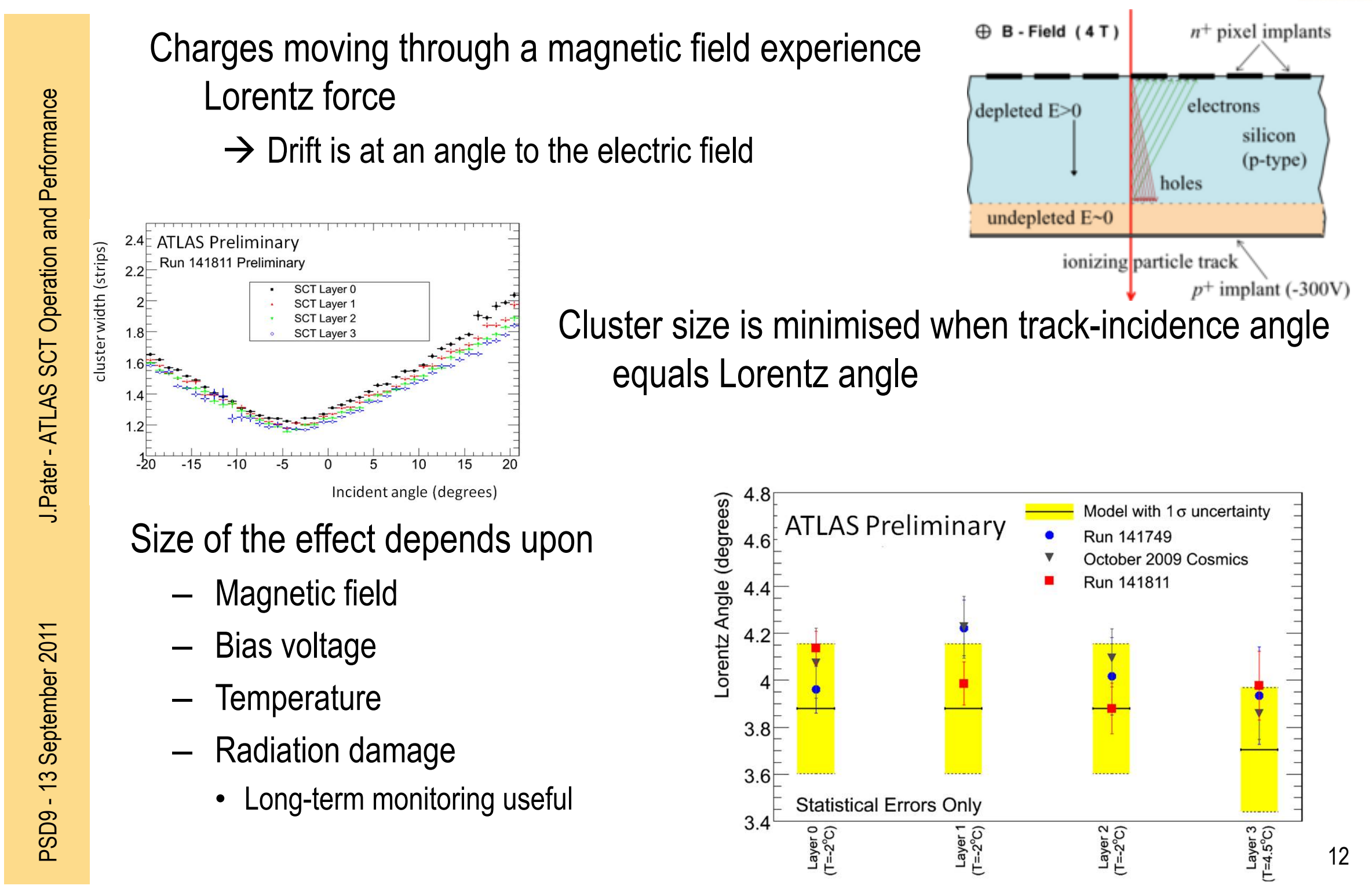




\section{Radiation Damage}

Must monitor and understand radiation damage for future

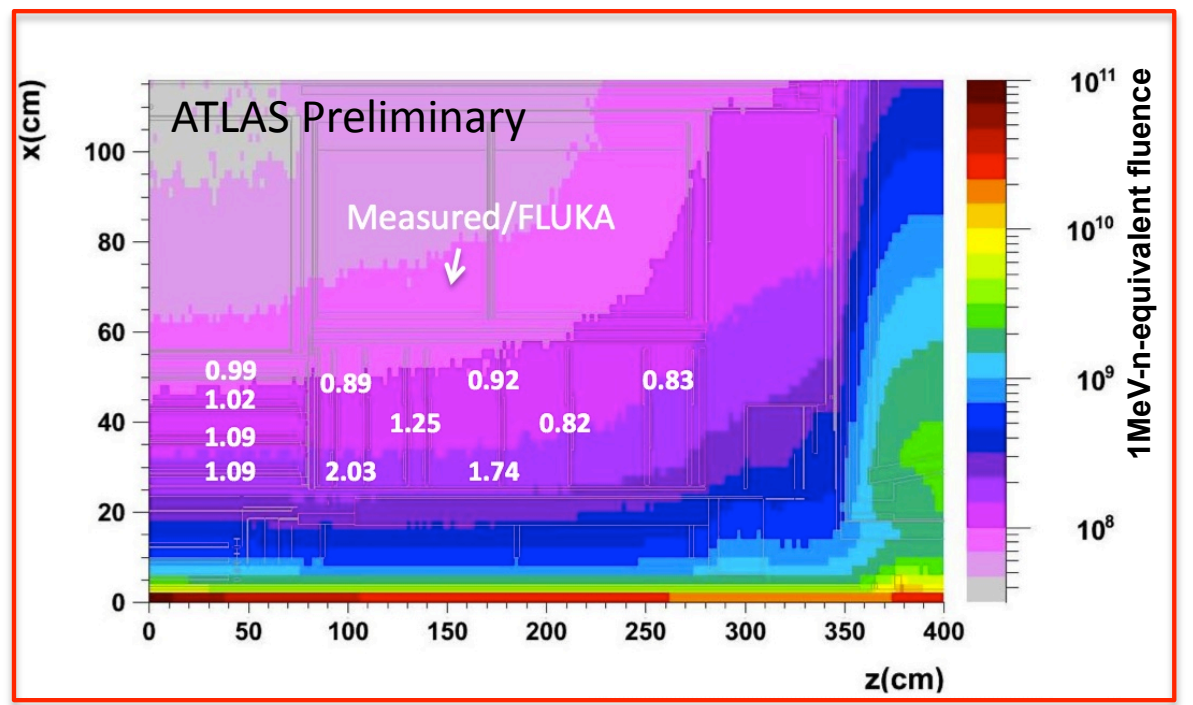

Comparisons with FLUKA predictions:

- Good in barrel region

- Discrepancies in low-R forward region

- Needs to be understood

\section{Leakage current is a good} indicator of radiation damage

- Measurements so far agree well with predictions

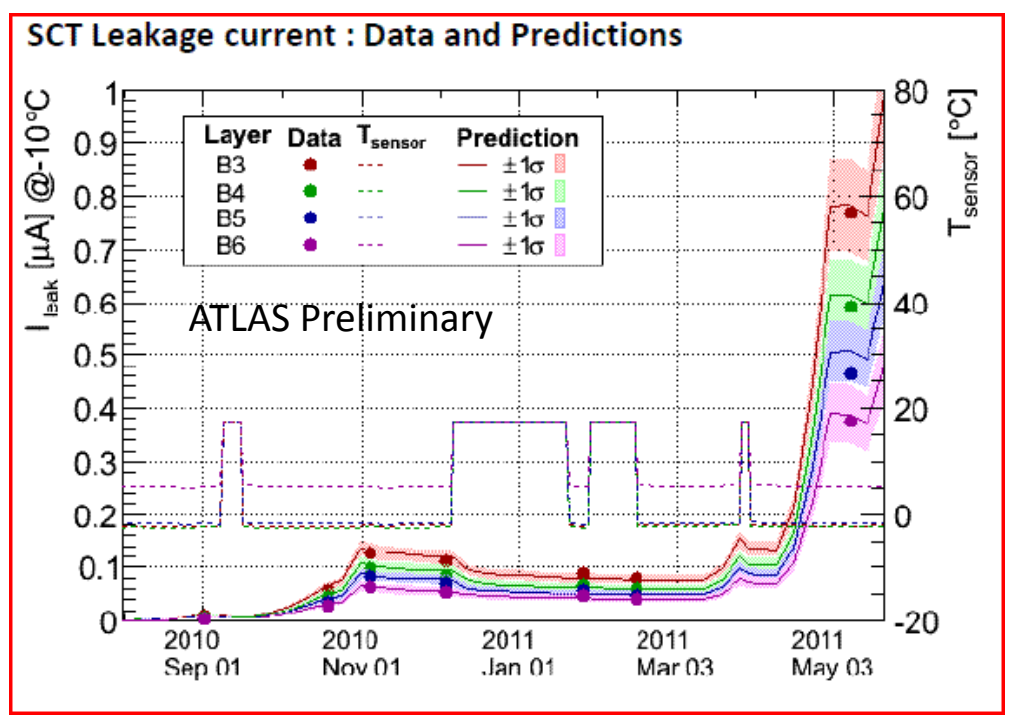




\section{Summary}

- ATLAS SCT is running very well:

- Stable

- Within design specifications

- Very low inefficiencies.

- Problems encountered with optical readout

- Spares and redundancy saved us!

- Radiation damage is being monitored and is in reasonable agreement with expectations.

- Looking forward to continued good performance and lots more data. 